\title{
Can extrapolation to the basis set limit be an alternative to the counterpoise correction? A study on the helium dimer
}

\author{
A. J. C. Varandas
}

Received: 14 December 2007 / Accepted: 4 February 2008 / Published online: 29 February 2008

(C) Springer-Verlag 2008

\begin{abstract}
Configuration interaction and coupled cluster calculations are reported for $\mathrm{He}_{2}$ using various orbital basis sets of the d-aug-AVXZ type, with the results being extrapolated to the one electron basis set limit both with counterpoise and without counterpoise correction. A generalized uniform singlet- and triplet-pair extrapolation scheme has been utilized for such a purpose. Using appropriate corrections to mimic full configuration interaction, the energies were predicted in excellent agreement with the best available estimates. The results also suggest that extrapolation to the complete basis set limit may be a general alternative to the counterpoise correction that yields a more accurate potential energy while being more economical.
\end{abstract}

\section{Introduction}

It is well known that the interaction energy of weakly bound species should be calculated with electronic structure methods that offer a balanced description of both the complex (supermolecule) and separated fragments. Stated concisely, one should warrant the correct dependence of the energy on the size of the system (size-extensivity) or, using superficially similar words, the correct separation of the molecule into its fragments. This is often referred as "size-consistency" $[1,2]$ to imply that the method when applied for electronic structure calculations on a diatomic $\mathrm{AB}$ gives $\lim _{R \rightarrow \infty} E_{\mathrm{AB}}=$ $E_{\mathrm{A}}+E_{\mathrm{B}}$. More generally, for a size-consistent method, the energy of a group of non-interacting molecules will equal the sum of the energies of each individual member of the group. Examples of such methods are the Hartree-Fock (HF),

A. J. C. Varandas $(\varangle)$

Departamento de Química, Universidade de Coimbra,

3004-535 Coimbra, Portugal

e-mail: varandas@qtvs1.qui.uc.pt full configuration interaction (FCI) and coupled cluster (CC) methods $[3,4]$. Of course, truncation of the single-reference $\mathrm{CI}$ expansion such as to include only single and double (SD) electronic excitations will not satisfy the size-consistency property since it retains unlinked diagram contributions [3, 4]. The same holds for the popular multi-reference CI approach including single and double electronic excitations (MRCISD or simply MRCI). However, when HF orbitals are used, the (single-reference) averaged coupled-pair functional (ACPF) method [5] that may be regarded as a modification of MRCI is size-extensive for a system composed of equal subsystems. As we will see in the present work, size-consistent methods are particularly valuable for studying closed-shell interactions, with $\mathrm{He}_{2}$ being a well studied prototype.

A second and particularly acute difficulty that arises in the calculation of weakly bound interactions is due to using finite sets of basis functions. The description of the complex and the fragments will then be unbalanced as an attractive interaction between the involved species will develop even in the absence of any interaction between the fragments. This spurious interaction is known as the basis set superposition error (BSSE), with much work having been devoted to its understanding over the years [4,6-11] (the list is by no means exhaustive). The most popular approach to account for BSSE is the counterpoise (CP) method of Boys and Bernardi [12], where the monomer energies are calculated with the full dimer basis. Although it has been objected that the monomers cannot take advantage of the full dimer basis as the Pauli principle prevents them from using the occupied orbitals of the partner $[13,14]$, the most popular avenue continues to be the full CP method [12]. Since BSSE is ubiquitous and general, it may seriously affect the weak interaction regions of any potential energy surface, be it for a closed-shell or open-shell interaction. In this regard, one may even wonder whether BSSE will get enhanced due to size-inconsistency of the methods 
employed for the calculation as the CI and MRCI approaches are possibly the most popular ones for studying open-shell interactions. As it will be shown later, the answer is negative when the configuration interaction method [without, CI, and with consideration of the popular Davidson correction for quadruple excitations, $\mathrm{CI}(\mathrm{Q})]$ is used for the title closedshell system. It will be also shown that the difficulties in calculating accurate potential energy surfaces can be minimized by extrapolating the raw energies without $\mathrm{CP}(\mathrm{NCP})$ to the complete basis set (CBS) limit. Although this may appear obvious as the BSSE vanishes by definition at the CBS limit, it is unclear whether CBS extrapolations of CP and NCP energies will lead to the same extrapolated value. From studies on five hydrogen-bonded complexes, Halkier et al. [7] noted that CP and NCP HF interaction energies both converge unsystematically, with a similar trend being observed for the NCP correlation contribution. This unsystematic behavior has been attributed to the fact that "the BSSE and the error from the incomplete description of the electronic Coulomb cusp both are present". They concluded [7] that once the former effect has been effectively removed by $\mathrm{CP}$, the cusp dominates and the convergence of the CP correlation contribution follows an $X^{-3}$ form similar to the form for the correlation energy (see Sect. 2.2). This suggests that an accurate description of weak interactions can only be done at the expenses of a huge computational effort since both $\mathrm{CP}$ and CBS treatments would be required. For two distinct fragments, it would amount to at least six molecular calculations per geometry. In fact, for many-body (fragment) systems, any BSSE treatment will be considerably more expensive and even hardly doable in a unique manner due to many-body effects [15-18]. Indeed, it is not clear whether two-body interactions in many-body systems should be computed in the basis set of the whole system or of the dimer. Moreover, $(n \geq 3)$-body effects are known to be considerably smaller than two-body ones, and hence a higher accuracy must be achieved in their calculation. Finally, existing formulations assume the Wigner-Witmer spin-spatial rules to be satisfied with one set of electronic states for the fragments over the whole configuration space, which is clearly not the general situation [19].

Although we shall not contest the view that accounting separately for both BSSE and CBS is the safe rule [7,9] for an accurate potential energy surface, we will investigate in the present work whether the situation can be ameliorated with somewhat more flexible basis sets and a recently proposed [20] extrapolation scheme. Indeed, Schwenke and Truhlar [21] have found earlier that the inclusion of the CP correction may not warrant by itself a systematic improvement of the calculations, and hence it is worth investigating whether an optimal solution for NCP extrapolation can still be found by using affordable basis sets and subsets of $X$ values. Part of the answer to our queries is implicit in the results of Ref. [7] where the singly diffusely augmented correlation-consistent polarized valence basis sets $[22,23]$ (aug-cc-pVXZ or simply AV $X Z$ ) with $X=D: 2, T: 3, \ldots, 5$ have been employed, but we will re-examine here the problem for the very weakly bound helium dimer with the doubly diffusely augmented basis set (d-aug-cc-AV $X \mathrm{Z}$ or d-AV $X \mathrm{Z}$ ) and $X$ values up to 6 . The results will be shown to be positive, as they suggest that CBS may to a large extent be all that is required. This implies that the BSSE extrapolates to a very small value (zero if the procedure was exact) as one could expect from being a basis set size effect, thus manifesting on the HF and correlation energies due to incompleteness of the one-electron basis set as for the incomplete description of the electronic Coulomb cusp. While noting the distinct physical origins of such effects [7], we will emphasize their coupling at the CBS limit in the sense that the BSSE will then vanish. In fact, the observed nonzero extrapolated-energy difference will help in defining the size of the error made upon CBS extrapolation.

Despite the fact that $\mathrm{He}_{2}$ has only four electrons, the accurate computation of a reliable potential for this system provides an enormous challenge in electronic structure theory when performed via main-stream electronic-structure methods of the CI type. This is because the interaction is weak $\left(D_{e} \sim 35 \mu \mathrm{E}_{\mathrm{h}}\right.$ ), and the dimer is only barely bound. Recently, the $\mathrm{He}_{2}$ dimer potential has been computed with the explicitly correlated multi-reference averaged coupledpair functional ( $\left.r_{12}-\mathrm{MR}-\mathrm{ACPF}\right)$ method [24], and with MRCI [25] and CC methods, including corrections for both the FCI and the basis set limit [26]. References [25] and [26] also include corrections for the basis set limit which have been taken from $\operatorname{CCSD}(\mathrm{T})-\mathrm{R} 12$ calculations $[27,28]$. In turn, calculations employing the quantum Monte Carlo [29,30] (QMC) approach and symmetry-adapted-perturbation theory [31] (SAPT) have also been performed. Moreover, Klopper [32] reported an accurate extrapolation of the $\mathrm{He}_{2}$ potential to the limit of a complete basis using CC methods, both including single and double excitations (CCSD [33]) as well as perturbative corrections for connected triple excitations [CCSD(T) [34]]. Most of such accurate works, either directly $[24,35,36]$ or indirectly (i.e., correcting the result for basis set unsaturation, as has been done in Refs. [25,26]) make use of explicitly correlated methods. More recently, calculations $[37,38]$ have been performed using supermolecular Gaussian geminal calculations. In these, the bulk of the interaction energy was estimated using the Gaussian geminal implementation of CC theory with double excitations (CCD), while the smaller contribution due to single, triple and quadruple excitations has been estimated using the conventional $\operatorname{CCSD}(\mathrm{T})$ and FCI methods. In the last publication, large atomic d-AVXZ basis sets with $X$ up to 6 have been utilized, and extrapolations are performed. In summary, some of the most accurate calculations reported thus far have employed explicitly correlated methods by using terms that are linear 
in the inter-electronic coordinates [24] $r_{i j}$, an approximation thereof [35], $r_{i j}=\sum_{\mu} \exp \left(\gamma_{\mu} r_{i j}^{2}\right)$, or explicitly correlated Gaussian geminals [37]. Indeed, the accuracy achieved is amazing, with the best binding energy estimates differing on small fractions of a kelvin and the most accurate interaction energy at a distance of $R=5.6 \mathrm{a}_{0}$ believed to be $-11.0037 \pm 0.0031 \mathrm{~K}[38]$.

The paper is organized as follows. In Sect. 2, we describe the procedure used to obtain the interaction potential of $\mathrm{He}_{2}$, while the results are reported and discussed in Sect. 3. The conclusions are in Sect. 4. For convenience, the calculated energies are given in micro-hartree, and the binding energies in kelvin $\left(1 \mu \mathrm{E}_{\mathrm{h}}=0.315775 \mathrm{~K}\right)$.

\section{Methodology}

\subsection{Basis-set superposition error and counterpoise}

Following common wisdom, we split the total energy into its $\mathrm{HF}$ and correlation (cor) components:

$$
E_{X}^{\mathrm{AB}}(R)=E_{X}^{\mathrm{AB}, \mathrm{HF}}(R)+E_{X}^{\mathrm{AB}, \text { cor }}(R)
$$

Traditionally, the interaction energy for an $\mathrm{AB}$ interaction will then assume the form

$$
\begin{aligned}
\Delta E_{X}^{\mathrm{AB}}(R) & =\sum_{\alpha=\mathrm{HF}, \mathrm{cor}} \Delta E_{X}^{\mathrm{AB}, \alpha}(R) \\
& =\sum_{\alpha=\mathrm{HF}, \text { cor }}\left[E_{X}^{\mathrm{AB}, \alpha}(R)-E_{X}^{\mathrm{A}, \alpha}-E_{X}^{\mathrm{B}, \alpha}\right]
\end{aligned}
$$

where the summation runs over the $\alpha=\mathrm{HF}$ and cor components, the fragment energies $E_{X}^{\mathrm{A}, \alpha}$ and $E_{X}^{\mathrm{B}, \alpha}$ are calculated in their own basis, and the energy of the complex, $E_{X}^{\mathrm{AB}, \alpha}(R)$, is calculated in the combined basis of the fragments. In turn, $X$ is a cardinal number that specifies a given basis in the hierarchical sequence of correlated-consistent ones [22,23] such as the double-augmented basis set (d-aug-cc-AVXZ or d-AVXZ) employed in the present study. ${ }^{1}$

As noted in Sect. 1, the traditional approach introduces a systematic error in the interaction energy, which is the more serious the poorer (smaller) the basis set $(X)$ is. In fact, during the calculation on the complex $\mathrm{AB}$, the description of fragment $\mathrm{A}$ is improved by the basis functions on $\mathrm{B}$ and vice-

\footnotetext{
${ }^{1}$ Basis sets were obtained from the extensible computational chemistry environment basis set database, version 02/25/04, as developed and distributed by the Molecular Science Computing Facility, Environmental and Molecular Sciences Laboratory which is part of the Pacific Northwest Laboratory, P.O. Box 999, Richland, Washington 99352, USA, and funded by the U.S. Department of Energy. The Pacific Northwest Laboratory is a multi-program laboratory operated by Battelle Memorial Institute for the U.S. Department of Energy under contract DE-AC06-76RLO 1830. Contact Karen Schuchardt for further information.
}

versa, while in the calculation of the fragment energies only their own basis sets are employed. As a result, the calculated interaction energy will contain, in addition to the true interaction energy, a spurious attractive contribution (BSSE) that arises from the improved description of the fragments in the improved basis. To account for this problem, Boys and Bernardi suggested to calculate the $\mathrm{CP}$-corrected interaction energy of $\mathrm{AB}$ as

$$
\begin{aligned}
\Delta E_{\mathrm{CP}, X}^{\mathrm{AB}}(R)= & \sum_{\alpha=\mathrm{HF}, \mathrm{cor}} \Delta E_{\mathrm{CP}, X}^{\mathrm{AB}, \alpha}(R)=\sum_{\alpha=\mathrm{HF}, \mathrm{cor}}\left[E_{X}^{\mathrm{AB}, \alpha}(R)\right. \\
& \left.-E_{X}^{\mathrm{AQ}, \alpha}(R)-E_{X}^{\mathrm{BQ}, \alpha}(R)\right]
\end{aligned}
$$

where $\mathrm{Q}$ is the dummy atom of $\mathrm{A}$ or $\mathrm{B}$. The $\mathrm{CP}$ correction is then given by

$$
\begin{aligned}
\epsilon_{X}^{\mathrm{AB}}(R)= & \sum_{\alpha=\mathrm{HF}, \mathrm{cor}}\left[\Delta E_{\mathrm{CP}, X}^{\mathrm{AB}, \alpha}(R)-\Delta E_{X}^{\mathrm{AB}, \alpha}(R)\right] \\
= & \sum_{\alpha=\mathrm{HF}, \mathrm{cor}}\left\{\left[E_{X}^{\mathrm{A}, \alpha}-E_{X}^{\mathrm{AQ}, \alpha}(R)\right]\right. \\
& \left.+\left[E_{X}^{\mathrm{B}, \alpha}-E_{X}^{\mathrm{BQ}, \alpha}(R)\right]\right\} \\
= & \sum_{\alpha=\mathrm{HF}, \mathrm{cor}}\left[\epsilon_{X}^{\mathrm{A}, \alpha}(R)+\epsilon_{X}^{\mathrm{B}, \alpha}(R)\right]
\end{aligned}
$$

For a variational wave function, the total $\mathrm{CP}$ correction must be positive as the largest unsigned energy will be obtained with the more flexible basis set that includes the basis of the dummy atom. On the other hand, due to size-extensivity of the HF method, one has $\epsilon_{X}^{\mathrm{A}, \mathrm{HF}}(R=\infty)=\epsilon_{X}^{\mathrm{B}, \mathrm{HF}}(R=\infty)=0$. Moreover, for a variational wave function, both terms in the summation (4) must obey the relations $E_{X}^{\mathrm{A}, \alpha} \geq E_{X}^{\mathrm{AQ}, \alpha}(R)$, and $E_{X}^{\mathrm{B}, \alpha} \geq E_{X}^{\mathrm{BQ}, \alpha}(R)$. For the $\mathrm{CP}$ potential to vanish at infinitely large distances, one has therefore to refer the interaction to the $\mathrm{CP}$ correlation energy at infinity, $\epsilon_{X}^{\mathrm{AB} \text {, cor }}(R=\infty)$.

The actual interaction energies to be extrapolated to $X=\infty$ are therefore $E_{X}^{\mathrm{AB}, \alpha}(R)$ in Eq. (2), the only one required for the CBS traditional interaction energy, and $E_{X}^{\mathrm{AQ}, \alpha}(R)$ and $E_{X}^{\mathrm{BQ}, \alpha}(R)$ when seeking to obtain the extrapolated $\mathrm{CP}$ interaction energy in Eq. (3). Rather than extrapolating separately the energy of the molecular complex and the energies of the fragments, one might hope to do instead an extrapolation of the interaction potential itself such as to reduce error-cancellation to a minimum level. Despite their great similarity, it turns out that there is no reason of principle to expect the diatomic to extrapolate for every value of $R$ as a fragment dressed with the ghost functions of the other. In particular, the extrapolation laws for the HF and cor components are known to have a distinct dependence on $X$, albeit the fact that the same rules have been employed for the total interaction energy [39]. We will therefore carry out separate extrapolations for the HF and cor components, as well as of 
the total interaction energy under the premise of dominance of the correlation character.

\subsection{Extrapolation methods}

Complete basis set extrapolations have been inferred from the dependence of the correlation energy on the partial wave quantum number for two-electron atomic systems and second-order pair energies in many-electron atoms [40-42]. The most popular two-parameter CBS scheme assumes the form [43]

$E_{X}^{\mathrm{cor}}=E_{\infty}^{\mathrm{cor}}+\frac{A_{3}}{(X+\alpha)^{3}}$

where $E_{X}^{\text {cor }}$ is the correlation energy for basis set of cardinal number $X$, and $E_{\infty}^{\text {cor }}$ and $A_{3}$ are parameters commonly determined from calculations for the two highest affordable values of $X ; \alpha$ is an offset parameter fixed from an auxiliary condition. For a recent analysis on the performance of Eq. (5) and a few other rules, see Ref. [44].

The uniform singlet- and triplet-pair extrapolation (USTE [20]) method (see also Ref. [45]) is based on the threeparameter rule

$E_{X}^{\mathrm{cor}}=E_{\infty}^{\mathrm{cor}}+\frac{A_{3}}{(X+\alpha)^{3}}+\frac{A_{5}}{(X+\alpha)^{5}}$

with $A_{5}$ defined by

$A_{5}=A_{5}^{o}+c A_{3}^{m}$

where $E_{\infty}^{\text {cor }}, A_{5}(0)=A_{5}^{o}, A_{3}, c$, and $\alpha$ are parameters. By fixing $\alpha, A_{5}^{o}, c$, and $m$ from other criteria, Eq. (6) has been transformed into an effective two-parameter $\left(E_{\infty}^{\text {cor }}, A_{3}\right)$ rule [20]. Using the USTE model, we have shown [46] that both the full correlation in systems studied by the popular singlereference Møller-Plesset (MP2) and coupled cluster [CCD and CCSD] methods as well as its dynamical part in MRCI(Q) calculations [20] or even correlation energies obtained by correlation energy extrapolation via intrinsic scaling [47] can be accurately extrapolated to the CBS limit. For the dynamical correlation, the optimum values of these parameters are [20] $A_{5}^{o}=0.003769$ and $c=-1.1784771 \mathrm{E}_{\mathrm{h}}^{-5 / 4}$, with $m=5 / 4$ and $\alpha=-3 / 8$. Corresponding parameters have been given for other methods with $\alpha=-3 / 8$, namely $A_{5}^{o}=0.1660699, c=-1.4222512 \mathrm{E}_{\mathrm{h}}^{-1}$, and $m=1$ for CC methods. Their extendibility ("universality") has also been explored by showing that they lead to accurate results for systems that were not part of the calibrating set $[46,48]$.

Figure 1 shows that the coefficients $\left(A_{5}, A_{3}\right)$ determined from the $\mathrm{CI}(\mathrm{Q}) / \mathrm{d}-\mathrm{AV} X \mathrm{Z}, \mathrm{CCSD} / \mathrm{d}-\mathrm{AV} X \mathrm{Z}$, and $\mathrm{CCSD}(\mathrm{T}) / \mathrm{d}-$ $\mathrm{AVXZ}$ calculations here reported are well described by Eq. (7). However, there may be difficulties close to the origin (small values of $A_{3}$ ) which correspond to small values of $A_{5}$. Since a recalibration of Eq. (7) can hardly be numerically
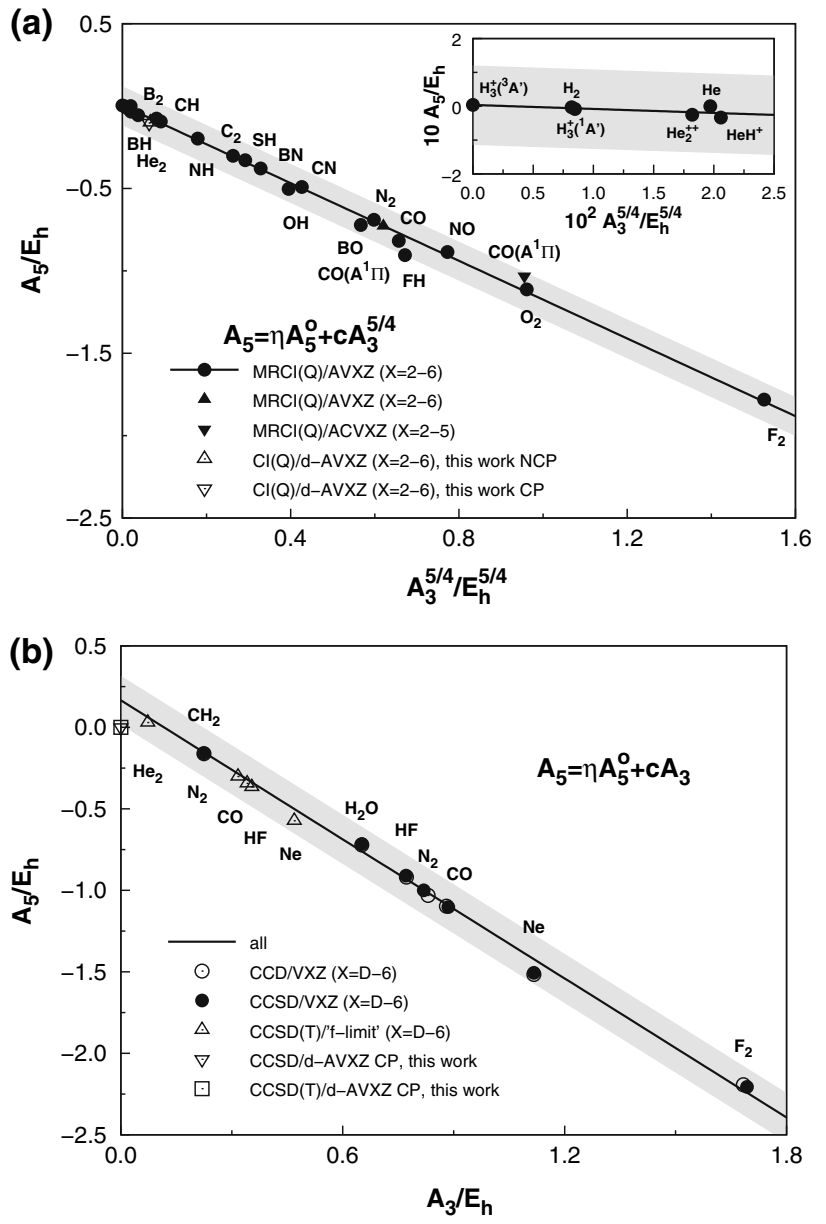

Fig. $1 A_{5}$ versus $A_{3}$ correlations [20] for various systems and correlated consistent orbital basis sets: a $\mathrm{CI}(\mathrm{Q})$ and $\mathrm{MRCI}(\mathrm{Q})$; b CCD, CCSD, and $\operatorname{CCSD}(\mathrm{T})$. The black solid dots in a refer to the calculations reported in Ref. [20], while the solid (up and down) triangles refer to the calculations [48] for the ground electronic and first-excited $\left(A^{1} \Pi\right)$ states of carbon monoxide. The CI(Q) energies that have been obtained in a similar manner for $\mathrm{He}_{2}$ both using NCP and CP methods are also shown by the up-triangle and down-triangle in $\mathbf{a}$. Similar trends are observed for the CCSD and CCSD(T) points of $\mathrm{He}_{2}$ which are presented only at the CP level. Also shown for completeness are other $\mathrm{CC}$ results, using a notation identical to that utilized elsewhere [20]. The shadowed areas have been obtained by moving the fitted [20,48] straight lines up and down such as to encompass all calculated points: $-0.113 \leq \eta A_{5}^{o} \leq 0.120$ for $\mathrm{CI}(\mathrm{Q}) ;-0.164 \leq \eta A_{5}^{o} \leq 0.096$ for CCSD and $\operatorname{CCSD}(\mathrm{T})$. See also the text

improved, we have adopted the following procedure. First, we have scaled the intersection of the fitted line at the origin by replacing $A_{5}^{o} \rightarrow \eta A_{5}^{o}$, with $\eta$ in Eq. (7) being chosen such as to impose the predicted $\tau_{53}=A_{5} / A_{3}$ ratio. This has been determined here from the average of the ratios calculated with $(D, T, Q)$ and $(D, T, Q, 5,6)$ basis sets. The result is $\tau_{53}=-0.96 \pm 0.04$ both for the CI(Q) and CC [CCSD and $\operatorname{CCSD}(\mathrm{T})]$ results. Although the scaling factor may vary with the pair (or range) of cardinal numbers used for the extrapolation, it has been found to be rather insensitive to $X$ for the 
title system, with the result being $\eta=0.30 \pm 0.02$. Interestingly, if Truhlar's protocol [49] $E_{X}=E_{\infty}+b / X^{2.4}$ [this has been optimized for $(D, T)$ extrapolations from CCSD and $\operatorname{CCSD}(\mathrm{T})$ energies] was employed to generate an extra point in the $D-T$ interval $(2 \leq X \leq 3)$, such a result could be utilized with the $X=D, T$ raw energies to get $\eta$ (jointly with $E_{\infty}$ and $A_{3}$ ) by fitting the three data points so obtained. The result in this case is $\eta \sim 0.34$, which is in good agreement with the value reported above. This would correspond to a value of $\tau_{53} \sim-0.89$ that nearly falls within the error limits above established from the raw ab initio energies. Unfortunately, no such a rule is available [49] for CI(Q) energies, although the above result seems to imply that it should be pretty much similar to the one for the CC family of methods. A final remark to note that the $\tau_{53}$ ratio for $\mathrm{He}_{2}$ lies close (but, as expected, is not identical) to the theoretical value [20] of -0.75 predicted from second-order MøllerPlesset perturbation-theory for natural-parity singlet states [41] in two-electron systems. Further details concerning the above generalized USTE (GUSTE) approach will be given elsewhere [50].

For the uncorrelated HF and CAS (uncorrelated in the sense of lacking dynamical correlation) energies, several schemes have been advanced ([20,51], and references therein). A popular rule is $E_{X}^{\mathrm{CAS}}=E_{\infty}^{\mathrm{CAS}}+A \exp (-b X)$, where $E_{\infty}^{\mathrm{CAS}}, A$, and $b$ are parameters to be determined from a fit to the raw HF energies, typically those calculated for the three or more highest affordable cardinal numbers. In [20], we have suggested a variant of such a rule based on fitting $X=T, Q, 5,6$ energies but with the recommended value obtained by averaging $E_{\infty}^{\mathrm{HF}}$ with the raw HF energy for the highest fitted value of $X$ (typically $X=6$ ). In addition, Karton and Martin [51] suggested the two-point extrapolation rule $A+B / X^{5.34}$ for $(T, Q)$ raw energies, while for the $(Q, 5)$ and $(5,6)$ ones they have recommended [51] $A+B(X+1) \exp (-9 \sqrt{X})$. Since the HF energies predicted from our own rule show generally good agreement with Karton and Martin [51] for the (5,6) case, we will adopt here for consistency both their formulas.

It should be noted that the extrapolations of $E_{X}^{\mathrm{HF}}$ and $E_{X}^{\text {cor }}$ are both geometry-dependent, and hence must be performed pointwise. Although the USTE [20] method combined with correlation scaling [52,53] has shown $[48,53]$ significant computer-time savings, this hybrid method will not be utilized such as to limit errors to a minimum level.

\section{Results and discussion}

In the case of two interacting He atoms, the raw energies to be extrapolated are $E_{\mathrm{CP}, X}^{\mathrm{He}_{2}, \mathrm{HF}}(R), E_{\mathrm{CP}, X}^{\mathrm{He}_{2}, \mathrm{cor}}(R), E_{X}^{\mathrm{HeQ}, \mathrm{HF}}(R)$, and $E_{X}^{\mathrm{HeQ}, \mathrm{cor}}(R)$. All such energies have been calculated using the MOLPRO suite of electronic structure programs [54].
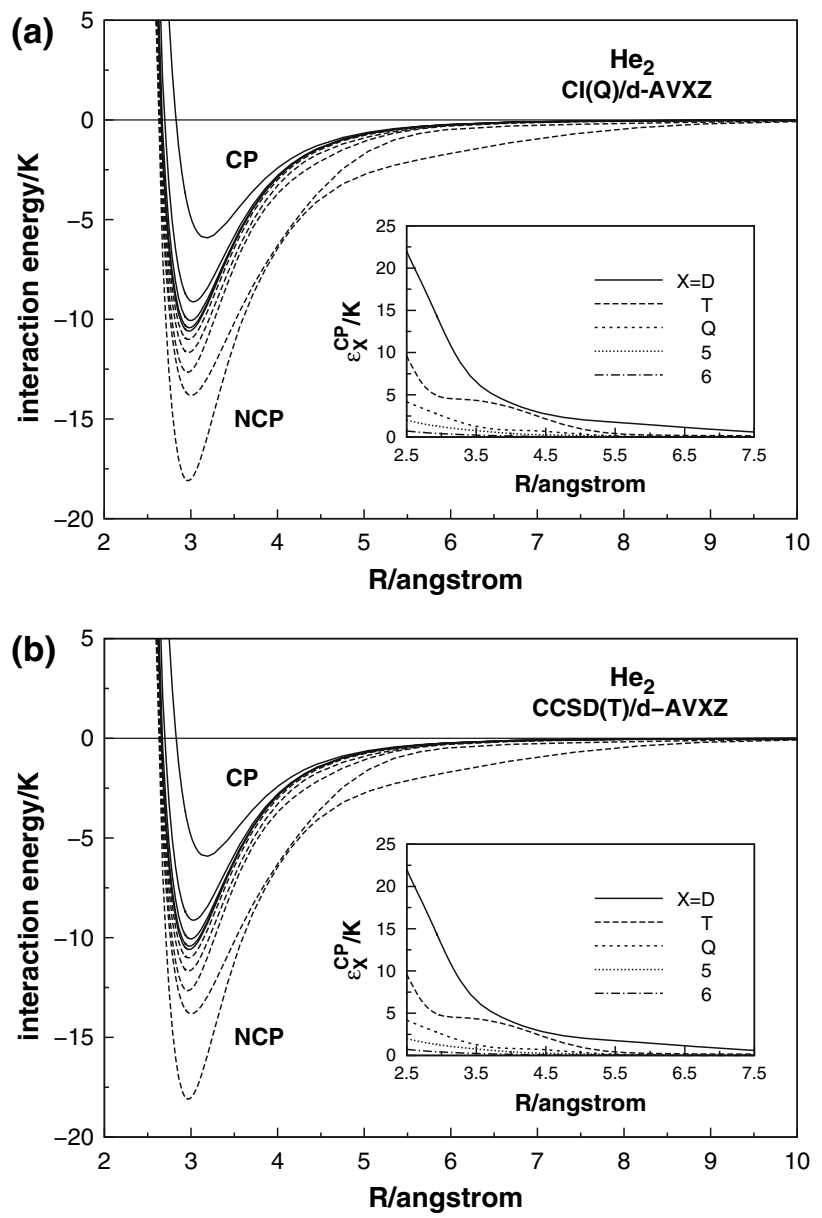

Fig. 2 Calculated raw interaction potentials for the helium dimer: a $\mathrm{CI}(\mathrm{Q}) / \mathrm{d}-\mathrm{AV} X \mathrm{Z} ; \mathbf{b} \mathrm{CCSD}(\mathrm{T}) / \mathrm{d}-\mathrm{AV} X \mathrm{Z}$. The solid lines refer to the potentials obtained with the $\mathrm{CP}$ method, while the NCP ones are indicated dashed lines. The NCP $(\mathrm{CP})$ potentials can be identified from $X=D$ to $X=6$ by moving upwards (downwards) at equilibrium

They correspond to the total $\mathrm{CP}$ and NCP interaction energies shown in panels (a) and (b) of Fig. 2a, b, respectively, for the $\mathrm{CI}(\mathrm{Q})$ and $\operatorname{CCSD}(\mathrm{T})$ methods. Note that the largest bond distance here considered of $R=297 \AA$ is assumed as infinitely large. For this, we obtain irrespectively of the cardinal number $\left|\epsilon_{X}^{\mathrm{He}, \mathrm{HF}}(R=297 \AA)\right| \leq 10^{-8} \mu \mathrm{E}_{\mathrm{h}}$ (the difference is on the last reported digit, which is negligibly small for the purpose of the present analysis), as expected due to the sizeextensivity of the HF method. Conversely, one gets for the d-AV DZ basis set using the CI method, $\epsilon_{D}^{\mathrm{He}, \mathrm{cor}}(R=297 \AA)=$ $276.20 \mu \mathrm{E}_{\mathrm{h}}$, while the corresponding result for the d-AV6Z basis set is $\epsilon_{6}^{\mathrm{He}, \text { cor }}(R=297 \AA)=329.18 \mu \mathrm{E}_{\mathrm{h}}$. However, if the calculations are carried with the popular Davidson correction for quadruple excitations $[\mathrm{CI}(\mathrm{Q})$, a notation that should not be confused with the commonly used $\mathrm{Q}$ to denote a ghost atom], the results are $\epsilon_{D}^{\mathrm{He}, \mathrm{cor}}(R=297 \AA)=-275.45 \mu \mathrm{E}_{\mathrm{h}}$ and $\epsilon_{6}^{\mathrm{He}, \mathrm{cor}}(R=297 \AA)=-327.04 \mu \mathrm{E}_{\mathrm{h}}$. Note that these $\mathrm{CI}(\mathrm{Q}) \mathrm{CP}$ corrections are negative which is likely due to 
Table 1 Extrapolated helium pair interaction potential at HF and CI levels in micro-hartree

\begin{tabular}{|c|c|c|c|c|c|c|c|c|c|c|c|c|c|}
\hline \multirow[t]{2}{*}{$R / \mathrm{a}_{0}$} & \multicolumn{6}{|l|}{$(T, Q)$} & \multicolumn{7}{|l|}{$(5,6)$} \\
\hline & $\Delta E_{\infty}^{\mathrm{HF}}$ & $\Delta E_{\mathrm{CP}, \infty}^{\mathrm{HF}}$ & $\epsilon_{\infty}^{\mathrm{HF}}$ & $\Delta E_{\infty}^{\mathrm{CI}(\mathrm{Q})^{\mathrm{a}}}$ & $\Delta E_{\mathrm{CP}, \infty}^{\mathrm{CI}(\mathrm{Q})}$ & $\epsilon_{\infty}^{\mathrm{CI}(\mathrm{Q})}$ & $\Delta E_{\infty}^{\mathrm{HF}^{\mathrm{b}}}$ & $\Delta E_{\infty}^{\mathrm{HF}}$ & $\Delta E_{\mathrm{CP}, \infty}^{\mathrm{HF}}$ & $\epsilon_{\infty}^{\mathrm{HF}}$ & $\Delta E_{\infty}^{\mathrm{CI}(\mathrm{Q})}$ & $\Delta E_{\mathrm{CP}, \infty}^{\mathrm{CI}(\mathrm{Q})}$ & $\epsilon_{\infty}^{\mathrm{CI}(\mathrm{Q})}$ \\
\hline 0 & $13,508.52$ & $13,517.51$ & -8.99 & 11970.41 & $11,967.25$ & 3.16 & $13,517.08$ & $13,517.50$ & $13,517.02$ & 0.49 & $11,996.12$ & $11,992.49$ & 3.63 \\
\hline 3.5 & $4,328.07$ & $4,334.32$ & -6.25 & $3,544.18$ & $3,537.42$ & 6.76 & $4,335.76$ & $4,336.27$ & $4,335.79$ & 0.48 & $3,555.98$ & $3,553.31$ & 2.66 \\
\hline 4.0 & $1,352.61$ & $1,357.81$ & -5.20 & 943.78 & 940.82 & 2.96 & $1,357.88$ & $1,358.24$ & $1,357.91$ & 0.33 & 947.38 & 946.33 & 1.06 \\
\hline 4.5 & 412.91 & 417.06 & -4.15 & 192.72 & 194.65 & -1.94 & 416.54 & 416.75 & 416.55 & 0.20 & 195.61 & 195.14 & 0.47 \\
\hline 5.0 & 121.47 & 125.92 & -4.45 & -0.78 & 4.17 & -4.95 & 125.55 & 125.72 & 125.53 & 0.19 & 4.26 & 3.74 & 0.52 \\
\hline 5.4 & 43.10 & 47.76 & -4.65 & -33.71 & -28.99 & -4.72 & 47.58 & 47.71 & 47.57 & 0.14 & -28.79 & -29.30 & 0.51 \\
\hline 5.6 & 24.98 & 29.33 & -4.36 & -35.90 & -32.15 & -3.75 & 29.20 & 29.31 & 29.19 & 0.12 & -31.86 & -32.30 & 0.44 \\
\hline 5.8 & 14.15 & 17.95 & -3.80 & -34.03 & -31.56 & -2.47 & 17.88 & 17.97 & 17.88 & 0.09 & -31.16 & -31.53 & 0.38 \\
\hline 6.0 & 7.80 & 10.9 & -3.18 & -30.31 & -29.10 & -1.21 & 10.94 & 11.00 & 10.93 & 0.08 & -28.66 & -29.00 & 0.34 \\
\hline 6.5 & 1.43 & 3. & -1.74 & -19.63 & -20.94 & 1.31 & 3.17 & 3.23 & 3.17 & 0.06 & -20.60 & -20.83 & 0.23 \\
\hline 7.0 & -0.02 & 0.91 & -0.93 & -11.62 & -14.12 & 2.50 & 0.92 & 0.98 & 0.91 & 0.07 & -13.89 & -14.02 & 0.14 \\
\hline 7.5 & -0.54 & 0.25 & -0.79 & -7.08 & -9.41 & 2.34 & 0.26 & 0.30 & 0.26 & 0.04 & -9.30 & -9.34 & 0.04 \\
\hline 8.0 & -0.92 & 0.08 & -1.00 & -4.82 & -6.36 & 1.54 & 0.08 & 0.07 & 0.08 & -0.01 & -6.26 & -6.19 & -0.07 \\
\hline
\end{tabular}

${ }^{a}$ In this table and Table 4, we use the usual notation $\mathrm{CI}(\mathrm{Q})$ to indicate that the CI energy includes the popular Davidson correction. This should not be confused with the use of $\mathrm{Q}$ to indicate a ghost atom

${ }^{\mathrm{b}}$ Numerical HF solution from Ref. [55]

the Davidson correction being non-variational, while the CI values are positive due to the variational nature of the calculation. Note further that the size of the BSSE can be an order of magnitude larger than the depth of the van der Waals well (see later) even for the d-AV6Z basis set, in agreement with similar findings reported [4] for other rare gas dimers.

Table 1 lists the extrapolated HF and CI(Q) energies for the helium dimer. The first observation (valid for all methods utilized here) is that the CP energies show more stable and smooth patterns when plotted against $X$ than the NCP ones. In particular, the excellent agreement observed between the CP extrapolated HF energies and the Kobus [55] numerical results obtained by the finite-differences method is striking. This manifests on the fact that the CP correction is always positive for the $(5,6)$ extrapolation, as if it were a true variational calculation (the small negative difference at $8 \mathrm{a}_{0}$ is probably of numerical origin). Yet, the negative $\mathrm{CP}$ corrections observed for the $(T, Q)$ extrapolations are of only a few $\mu \mathrm{E}_{\mathrm{h}}$, and hence may be tolerable for many situations of practical interest. We further observe that the largest $\mathrm{CP}$ correction arises in the correlation energy term, with the corresponding $\mathrm{HF}$ contribution in the $(5,6)$ extrapolation being always smaller than $0.5 \mu \mathrm{E}_{\mathrm{h}}$. Finally, and not surprising, the $(5,6)$ extrapolated $\mathrm{CI}(\mathrm{Q})$ binding energy at $5.6 \mathrm{a}_{0}$ is $10.20 \mathrm{~K}$, which is $\sim 0.80 \mathrm{~K}$ smaller than the best available estimates. This is mostly due to the missing triple (and approximated quadruple) electron excitations in the raw $\mathrm{CI}(\mathrm{Q})$ energies.

The extrapolated $\mathrm{He}_{2}$ interaction potentials obtained from the CCSD/d-AVXZ and CCSD(T)/d-AVXZ calculations here reported are listed in Table 2 . We first note that the
CP correction for a given $\left(X_{1}, X_{2}\right)$ cardinal-number pair is identical for the CCSD and $\operatorname{CCSD}(\mathrm{T})$ energies. This can be understood from Eq. (4) which shows that $\epsilon_{X}^{\mathrm{AB}}$ depends only on the energy of the monomers [which is identical for both the CCSD and CCSD(T) methods due to the absence of electron excitations higher than doubles]. We emphasize that the $\mathrm{CP}$ correction for the $(5,6)$ extrapolations is small and positive for all distances. Moreover, the binding energy at $5.6 \mathrm{a}_{0}$ is $10.80 \mathrm{~K}$, thus larger by $0.60 \mathrm{~K}$ than the $\mathrm{CI}(\mathrm{Q})$ value, which is partly due to the size-consistency of the $\operatorname{CCSD}(\mathrm{T})$ method. We further observe that the $(5,6)$ CCSD binding energy at $5.6 \mathrm{a}_{0}$ using $\mathrm{NCP}(\mathrm{CP})$ is $1.86 \mathrm{~K}(1.86 \mathrm{~K})$ smaller than the $\mathrm{CCSD}(\mathrm{T})$ one, which highlights the importance of the perturbative correction for the triple electron excitations. Finally, the $(5,6)$ NCP binding energy is seen to underestimate the $\mathrm{CP}$ one, with an opposite trend holding for the $(T, Q)$ extrapolation. This may be explained from the rough variation of the NCP results with $X$. In fact, Fig. 3 shows that the $(D-Q)$ extrapolation of the total interaction energy via Eqs. (6 and 7) (with $\eta A_{5}^{o}$ ) under the assumption of dominance of the electron correlation cannot be made without a sizable error. Also shown is the fact that the $\mathrm{CP}$ and NCP energies tend to converge to the basis set limit from each side, thus corroborating the findings reported in Ref. [7] for hydrogen-bonded systems. Such a pattern may vary though with the interatomic bond distance, with both curves approaching from above, e.g., for $R=4 a_{0}$.

Shown in the insets of Fig. 2 are the CP corrections for the $\mathrm{CI}(\mathrm{Q})$ (Fig. 2a) and $\operatorname{CCSD}(\mathrm{T})$ (Fig. 2b) methods. As shown, they look very much the same for a given value of $X$. 

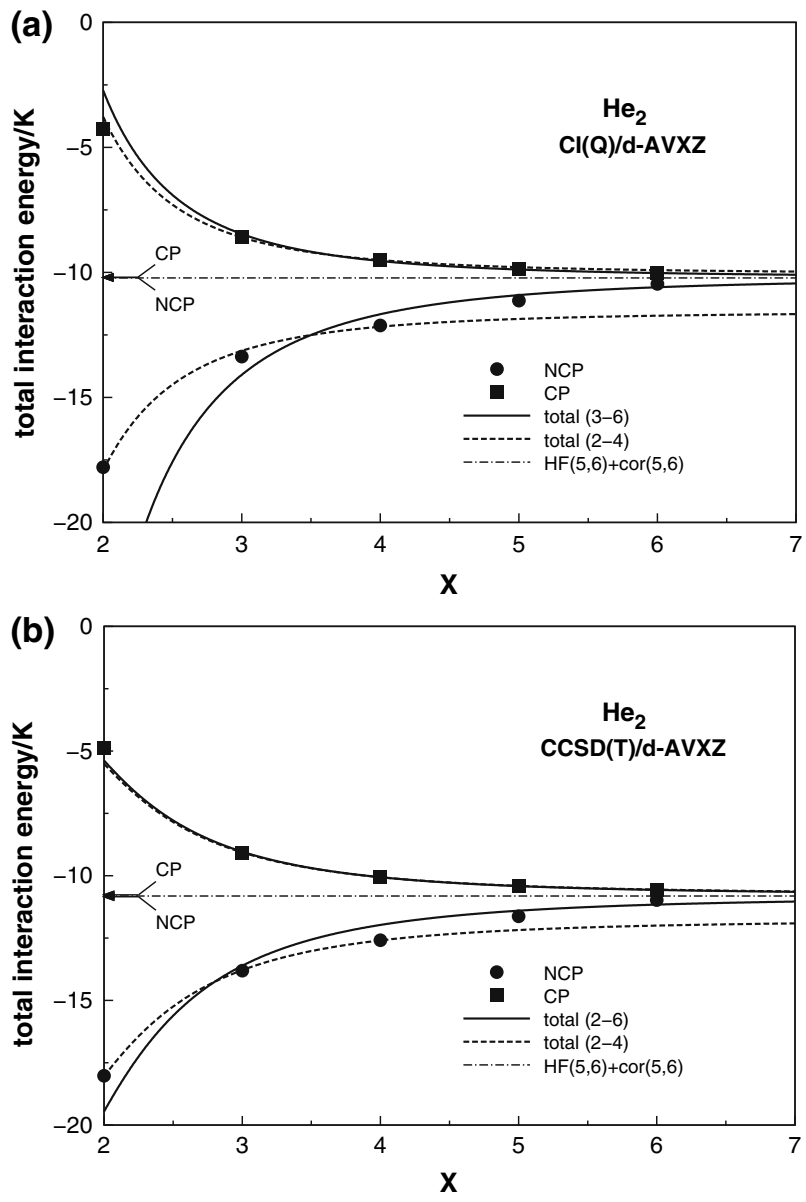

Fig. 3 Extrapolation of the raw total interaction energies of helium dimer at $5.6 \mathrm{a}_{0}$ : a $\mathrm{CI}(\mathrm{Q}) / \mathrm{d}-\mathrm{AV} X \mathrm{Z} ; \mathbf{b} \mathrm{CCSD}(\mathrm{T}) / \mathrm{d}-\mathrm{AV} X \mathrm{Z}$. Threeparameter fits for $X=D-Q$ (dashed lines) and $X=D-6$ or $X=T-6$ (solid lines) using the GUSTE model are also shown. The horizontal dash-dotted line indicates the result obtained from separate extrapolations of the HF and cor energies, while the asymptotes of both solid lines are indicated by arrows pointing to the left-hand-side axis. See also the text

In fact, the two $\mathrm{CP}$ corrections are indistinguishable to less than $3 \times 10^{-4} \mathrm{~K}$, and often to a much higher accuracy. This demonstrates that the BSSE does not seem to be influenced by the size-inconsistency of the $\mathrm{CI}(\mathrm{Q})$ method. As expected, the $\mathrm{CP}$ corrections (relative to the asymptote) are predicted to decrease sharply with $X$, being of subkelvin size beyond $X=6$. As shown from Tables 1 and 2 , such a pattern extends to the $\mathrm{CI}(\mathrm{Q})$ and $\operatorname{CCSD}(\mathrm{T})$ extrapolated energies, with the difference between $\epsilon_{\infty}^{\mathrm{CI}(\mathrm{Q})}$ and $\epsilon_{\infty}^{\mathrm{CCSD}(\mathrm{T})}$ being typically of a few $10^{-2} \mathrm{~K}$ and always smaller than $0.1 \mathrm{~K}$, which is most likely due to the numerical noise in the involved extrapolation procedures.

We now turn to a comparison with the result that is obtained when the traditional scheme $(5)$ using the $(5,6)$ cardinal numbers is used to extrapolate the correlation energy. Although the unsigned value so predicted underestimates the corresponding GUSTE result by $5.77 \mathrm{~K}$ at $5.6 \mathrm{a}_{0}$, the diffe- rence in interaction correlation energy (once subtracted the asymptote) is more attractive by $0.009 \mathrm{~K}$. Parenthetically, we mention that the results here reported for the $\operatorname{GUSTE}(5,6)$ interaction energies are essentially indistinguishable from those obtained from the original $\operatorname{USTE}(5,6)[20]$ method. One then expects the calculated total interaction energy based on Eq. (5) to agree within $0.01 \mathrm{~K}$ or so with the GUSTE prediction from the present work. On the other hand, since the $\mathrm{CP}$ interaction HF energy from Table 1 reproduces the value tabulated by Klopper [32] within its reported accuracy, this may explain the excellent agreement with the results obtained from Eqs. (5) and (9) of Ref. [32].

Before comparing with experiment, we follow Klopper [32] by noting that MRCI calculations in the IO301 basis [25], FCI calculations in the $\mathrm{AV} Q \mathrm{Z}$ and d-AVTZ basis [26], MR-ACPF calculations in the d-AV6Z basis [56], and the Gaussian geminal implementation of CCSD theory including effects of triple and quadruple excitations [37] (and employing the conventional orbital approach and very large augmented correlation-consistent basis sets extended by sets of bond functions) indicate that the step from $\operatorname{CCSD}(\mathrm{T})$ to FCI adds $\delta E_{(\mathrm{T}) \rightarrow \mathrm{FCI}}=-(0.323 \pm 0.005) \mathrm{K}$ to the well depth. Using even larger basis sets and extrapolations, this correction has most recently been slightly reduced to the value of [38] $\delta E_{(\mathrm{T}) \rightarrow \mathrm{FCI}}=-\left(0.318_{3} \pm 0.002_{8}\right) \mathrm{K}$, which will be utilized in the present work (for clarity, rather than rounding-off the number, we give heretofore the last digit as a sub-index). This leads to a binding energy of the extrapolated counterpoise $\operatorname{CCSD}(\mathrm{T})$ curve at $5.6 \mathrm{a}_{0}$ of $-11.11_{8} \mathrm{~K}$. Moreover, from our dense grid of points, the extrapolated $\operatorname{CCSD}(\mathrm{T})$ well depth with CP is $-34.25 \mu \mathrm{E}_{\mathrm{h}}=-10.82 \mathrm{~K}$ [or $-11.13_{8} \mathrm{~K}$ upon addition of $\left.\delta E_{(\mathrm{T}) \rightarrow \mathrm{FCI}}\right]$ at $2.7000 \pm 0.0005 \AA$.

When attempting to draw precise quantitative conclusions that amount to fractions of a kelvin, one may have to dwell for further accuracy (thus narrowing of the above uncertainty) by extrapolating from even larger cardinal numbers. From his CCSD(T) calculations, Klopper [32] has observed that going from a $(5,6)$ to $(6,7)$ extrapolation leads to a decrease of $0.09 \pm 0.04 \mathrm{~K}$ on the binding energy at $5.6 \mathrm{a}_{0}$, depending on the extrapolation law that is utilized. Since the $(5,6)$ extrapolations based on Eqs. (5) and (9) of Ref. [32] lead to the same result as the $\operatorname{GUSTE}(5,6)$ approach, we adopt the corresponding decrease of $0.115 \pm 0.005 \mathrm{~K}$ taken from Table III of that work. Addition of this refinement leads to our best prediction of $-11.00_{3} \mathrm{~K}$ at $5.6 \mathrm{a}_{0}$ or $D_{e}=-11.02_{3} \mathrm{~K}$. If one adopts instead the values obtained by direct extrapolation of the total $\operatorname{CCSD}(\mathrm{T})$ interaction energy using Eqs. (6) and (7) for $X=D-6$ (thus with GUSTE, i.e., assuming the dominance of the correlation energy), the result is $-10.80 \pm 0.03 \mathrm{~K}\left[-11.00_{3} \pm\right.$ $0.03 \mathrm{~K}$ upon addition of the above $\delta E_{(\mathrm{T}) \rightarrow \mathrm{FCI}}$ and $(6,7)$ corrections] at $5.6 \mathrm{a}_{0}$. Note that we have used the $\mathrm{CP}$ and $\mathrm{NCP}$ asymptotes to define the best value as their unweighted 
Table 2 Extrapolated helium pair interaction potential at CCSD and CCSD(T) levels in micro-hartree

\begin{tabular}{|c|c|c|c|c|c|c|c|c|c|c|}
\hline \multirow[t]{2}{*}{$R / \mathrm{a}_{0}$} & \multicolumn{5}{|l|}{$(T, Q)$} & \multicolumn{5}{|l|}{$(5,6)$} \\
\hline & $\Delta E_{\infty}^{\mathrm{CCSD}}$ & $\Delta E_{\mathrm{CP}, \infty}^{\mathrm{CCSD}}$ & $\Delta E_{\infty}^{\mathrm{CCSD}(\mathrm{T})}$ & $\Delta E_{\mathrm{CP}, \infty}^{\mathrm{CCSD}(\mathrm{T})}$ & $\epsilon_{\infty}^{\operatorname{CCSD}(\mathrm{T})}$ & $\Delta E_{\infty}^{\mathrm{CCSD}}$ & $\Delta E_{\mathrm{CP}, \infty}^{\mathrm{CCSD}}$ & $\Delta E_{\infty}^{\mathrm{CCSD}(\mathrm{T})}$ & $\Delta E_{\mathrm{CP}, \infty}^{\mathrm{CCSD}(\mathrm{T})}$ & $\epsilon_{\infty}^{\operatorname{CCSD}(\mathrm{T})}$ \\
\hline 3.0 & $12,029.38$ & $12,026.23$ & $11,920.91$ & $11,917.77$ & 3.15 & $12,053.85$ & $12,050.13$ & $11,948.16$ & $11,944.44$ & 3.73 \\
\hline 3.5 & $3,577.75$ & $3,570.99$ & $3,515.73$ & $3,508.97$ & 6.76 & $3,589.00$ & $3,586.28$ & $3,528.63$ & $3,525.92$ & 2.72 \\
\hline 4.0 & 962.58 & 959.57 & 928.61 & 925.60 & 3.01 & 966.05 & 964.91 & 932.79 & 931.65 & 1.14 \\
\hline 4.5 & 203.20 & 205.09 & 184.93 & 186.81 & -1.89 & 206.09 & 205.54 & 188.01 & 187.46 & 0.56 \\
\hline 5.0 & 5.10 & 10.00 & -4.80 & 0.10 & -4.90 & 10.16 & 9.56 & 0.33 & -0.28 & 0.61 \\
\hline 5.4 & -29.99 & -25.33 & -36.16 & -31.51 & -4.66 & -25.04 & -25.61 & -31.17 & -31.74 & 0.57 \\
\hline 5.6 & -32.91 & -29.21 & -37.81 & -34.11 & -3.70 & -28.84 & -29.31 & -33.72 & -34.20 & 0.48 \\
\hline 5.8 & -31.64 & -29.13 & -35.56 & -33.06 & -2.51 & -28.72 & -29.16 & -32.62 & -33.06 & 0.44 \\
\hline 6.0 & -28.38 & -27.13 & -31.53 & -30.29 & -1.24 & -26.69 & -27.10 & -29.81 & -30.22 & 0.41 \\
\hline 6.5 & -18.47 & -19.74 & -20.36 & -21.64 & 1.28 & -19.38 & -19.68 & -21.23 & -21.53 & 0.30 \\
\hline 7.0 & -10.89 & -13.36 & -12.05 & -14.52 & 2.46 & -13.10 & -13.27 & -14.25 & -14.43 & 0.18 \\
\hline 7.5 & -6.60 & -8.88 & -7.35 & -9.64 & 2.29 & -8.78 & -8.88 & -9.52 & -9.62 & 0.10 \\
\hline 8.0 & -4.49 & -5.98 & -4.98 & -6.48 & 1.49 & -5.91 & -6.03 & -6.39 & -6.51 & 0.12 \\
\hline
\end{tabular}

${ }^{\text {a }}$ Identical for both CCSD and CCSD(T) methods at a given $\left(X_{1}, X_{2}\right)$ extrapolation level

average. Similarly, the uncertainty has been defined such as to embrace such estimates. Tight error margins are also obtained for the total $\mathrm{CI}(\mathrm{Q})$ interaction energies, yielding an uncorrected binding energy of $-10.20 \pm 0.005 \mathrm{~K}$ at the same distance [note that we have preferred a (3-6) extrapolation as the (2-6) one extrapolates poorly in the NCP case: the asymptote underestimates slightly the last fitted raw energy]. Given the excellent though somewhat surprising (due to the approximations involved) agreement with the average values obtained above via separate extrapolations of the HF and cor energies, we will adopt the same uncertainty for the energies calculated by both methods. Similarly good agreement is obtained for 4 and $7 \mathrm{a}_{0}$, although the fitted range of $X$ values shows a slight influence on the final values (yet within the reported error bars). Clearly, as shown in Table 3, our CCSD(T) results extrapolated by the GUSTE method predict a well depth in very good agreement with Cencek et al. [37] at the three distances reported in their work. The agreement is even slightly better with the new result of Patkowski et al. [38] for 5.6 $\mathrm{a}_{0}$. Needless to say, at such a level of accuracy, relativistic and even QED corrections not to mention non-adiabatic effects will probably exceed the errors in the Born-Oppenheimer curve and hence should not be ignored. This is, however, outside the scope of the present work.

We conclude this section with some remarks on NCP and CP methods. Although CP energies usually facilitate extrapolation, Table 4 shows that the method can be rather expensive as it demands separate calculations on the dimer and monomers with the same basis set. Note that the latter may turn out to be somewhat more expensive than the former due to loss of symmetry. This may explain why $\mathrm{CP}$ is seldom used in detailed calculations of $N$-atom potential energy surfaces since the number of studied geometries scales as the power $3 N-6$ of the number of points required per dimension. For a homonuclear such as $\mathrm{He}_{2}$, the CPU time scales roughly as twice the time of a single calculation on the complex, while for an heteronuclear system $\mathrm{AB}$ a factor of threefold will generally apply. On the other hand, NCP or CP extrapolations must lead to the same result given increasingly large basis sets. This may be seen from the results here reported for $\mathrm{He}_{2}$, which may typify the trends to be expected on small molecular systems. A CP calculation using the CCSD(T) method predicts with a d-AV6Z basis set a binding energy of $-10.58 \mathrm{~K}$, while Table 2 and Fig. 4 show that a similar accuracy is achieved at a lower cost via $(T, Q)$ or $(5,6)$ extrapolations. For example, the NCP binding energy of $\mathrm{He}_{2}$ at $5.6 \mathrm{a}_{0}$ obtained from raw $\mathrm{CCSD}(\mathrm{T}) / \mathrm{AV} Q Z$ calculations is $-11.00 \mathrm{~K}$ but only $-4.29 \mathrm{~K}$ with $\mathrm{CP}$. These compare with the $(T, Q)$ extrapolated $\operatorname{CCSD}(\mathrm{T})$ values of $-11.89 \mathrm{~K}$ (NCP) and $-10.83 \mathrm{~K}(\mathrm{CP})$. In turn, the NCP result with a CCSD(T)/AV6Z basis set is $-10.40 \mathrm{~K}(\mathrm{NCP})$, while the $(5,6)$ values are $-10.64 \mathrm{~K}(\mathrm{NCP})$ and $-10.80 \mathrm{~K}(\mathrm{CP})$. Note that we are not adding $\delta E_{(\mathrm{T}) \rightarrow \mathrm{FCI}}$ to the values reported in this paragraph. Thus, with the exception of the NCP estimate with CCSD(T)/AV QZ where the good agreement with the best available estimates may have been accidental, the extrapolated $\left(X_{1}, X_{2}\right)$ results $(\mathrm{NCP}$ and $\mathrm{CP})$ turn out to have an enhanced quality when compared with the raw $\mathrm{CP}$ results for the basis set of highest cardinal number $\left(X_{2}\right)$.

The poor quality of the CCSD(T)/d-AVTZ results at NCP may explain the fluctuations in the $\operatorname{CCSD}(\mathrm{T}) / \mathrm{d}-\mathrm{AV}(T, Q) \mathrm{Z}$ curve, thus contrasting with the stable pattern of the corresponding $\mathrm{CP}$ results. This may explain the small deviations between the d-AV $(T, Q) \mathrm{Z}$ and d-AV $(5,6) \mathrm{Z}$ extrapolated $\mathrm{CP}$ 
Table 3 Interaction energies (in kelvin) for the helium dimer at three bond distances

\begin{tabular}{llll}
\hline Method & $R=4.0 \mathrm{a}_{0}$ & $5.6 \mathrm{a}_{0}$ & $7.0 \mathrm{a}_{0}$ \\
\hline Korona et al. $^{\mathrm{a}}$ & $291.64 \pm 0.87$ & $-11.059 \pm 0.03$ & $-4.629 \pm 0.03$ \\
Jeziorska et al. $^{\mathrm{b}}$ & & $-11.000 \pm 0.01$ & -10.798 \\
Komasa $^{\mathrm{c}}$ & 292.784 & -10.981 & $-10.98 \pm 0.02$ \\
Komasa $^{\mathrm{d}}$ & & $-10.998 \pm 0.005$ \\
Anderson $^{\mathrm{e}}$ & & $-11.004 \pm 0.03$ \\
Anderson $^{\mathrm{f}}$ & & $-10.99 \pm 0.02$ & $-10.980 \pm 0.004$ \\
van Mourik and Dunning $^{\mathrm{g}}$ & 293.496 & $-10.99 \pm 0.02$ & $-4.620 \pm 0.002$ \\
van de Bovenkamp and van Duijnveldt $^{\mathrm{h}}$ & $292.72 \pm 0.2$ & $-11.009 \pm 0.008$ & $-11.003_{7} \pm 0.003_{1}$ \\
Gdanitz $^{\mathrm{i}}$ & $292.75 \pm 0.01$ & $-11.00_{3} \pm 0.03$ & $-4.619 \pm 0.007$ \\
Klopper $^{\mathrm{j}}$ & $292.6 \pm 0.3$ & $-11.00_{3} \pm 0.03$ \\
Cencek et al. $^{\mathrm{k}}$ & $292.54 \pm 0.04$ & $-4.649 \pm 0.02$ \\
Patkowski et al. $^{1}$ & & $292.2_{0} \pm 0.4$ & $-4.63_{7} \pm 0.02$ \\
This work $^{\mathrm{m}}$ & $292.3_{1} \pm 0.4$ & \\
This work $^{\mathrm{n}}$ & &
\end{tabular}

${ }^{\text {a }}$ From symmetry adapted perturbation theory [31]

${ }^{\mathrm{b}}$ From symmetry adapted perturbation theory [57]

${ }^{c}$ Variational upper bounds using exponentially correlated Gaussian functions [35]

${ }^{\mathrm{d}}$ Variational upper bound [36]

${ }^{\mathrm{e}}$ From Quantum Monte Carlo [29] calculations

${ }^{\mathrm{f}}$ From Quantum Monte Carlo [30] calculations

g Extrapolated CCSD(T) values obtained by combining earlier results with non-extrapolated CCSDT and FCI energies [26]

${ }^{\mathrm{h}} \mathrm{MRCI}$ results from Ref. [25]

${ }^{\mathrm{i}}$ From $r_{12}$-MR-ACPF calculations [24]

${ }^{\mathrm{j}}$ Extrapolated results from $\operatorname{CCSD}(\mathrm{T})$ and FCI calculations in orbital bases [32]

${ }^{k}$ From supermolecular Gaussian geminal calculations [37]

${ }^{1}$ From supermolecular Gaussian geminal calculations [38]

${ }^{m}$ From this work by direct extrapolation of the total interaction energy using Eqs. (6) and (7) and the corrections to FCI from Ref. [38]: $\delta E_{(\mathrm{T}) \rightarrow \mathrm{FCI}}=-\left(1.88_{3} \pm 0.01_{1}\right) \mathrm{K}$ at $4 \mathrm{a}_{0} ; \delta E_{(\mathrm{T}) \rightarrow \mathrm{FCI}}=-\left(0.0808_{4} \pm 0.0003_{1}\right) \mathrm{K}$ at $7 \mathrm{a}_{0}$; see also text

${ }^{n}$ Result obtained from separate extrapolations of the NCP and CP raw CCSD(T) energies with the GUSTE(5, 6) method [20]. Results corrected for FCI as in footnote $m$ )

binding energies. Moreover, the shape of the d-AVDZ and d-AVTZ curves suggests that an improved NCP result may be obtained from $(D-Q)$ or $(D, Q)$ extrapolations, although this could somewhat be accidental too. However, the $(Q, 5)$ NCP extrapolation is seen from Fig. 4 to yield flatter energy differences as one would hope for use in spectroscopic calculations. Table 1 further suggests that the problem with the $(T, Q)$ extrapolation lies to a large extent on the HF energy as the corresponding $(T, Q)$ and $(5,6)$ interaction energies show marked differences. Unfortunately, this cannot be tested due to lack of precise $(D-Q)$ or $(D, Q)$ rules to extrapolate the HF energy. However, one may decide to converge the (easier to converge from the point of view of memory requirements but still time consuming; see Table 4) HF energy and combine the result with the correlation energy extrapolated from small- $X$ schemes. In this case, the difference between the NCP and CP total interaction energies equals the difference in the correlation energy components. For the
NCP extrapolation with $(D-Q)$ it amounts to $\sim-0.5 \mathrm{~K}$ [the minus sign implies overestimation of the $(5,6) \mathrm{CP}$ result], and $\sim 0.1 \mathrm{~K}$ for $(T, Q)$. This is roughly an order of magnitude smaller than the difference obtained $(1.4 \mathrm{~K})$ when the HF energy is similarly extrapolated.

Finally, a $\operatorname{CCSD}(\mathrm{T}) / \mathrm{d}-\mathrm{AV}(5,6) \mathrm{Z}$ calculation without $\mathrm{CP}$ takes CPU times roughly a factor of two smaller than a raw $\mathrm{CP}$ calculation with the $\mathrm{d}-\mathrm{AV} 6 \mathrm{Z}$ basis set. If one considers instead a $\operatorname{CCSD}(\mathrm{T}) / \mathrm{d}-\mathrm{AV}(T, Q) \mathrm{Z}$ extrapolation with $\mathrm{NCP}$, the scaling factor raises to 18 and over 100 when compared with raw CP calculations employing d-AV5Z and d-AV6Z basis sets, respectively. Hopefully, more reliable estimates can be obtained if extrapolating with $\mathrm{CP}$ energies, and this can still be done at a reasonable cost if based on $\operatorname{CCSD}(\mathrm{T}) / \mathrm{d}-\mathrm{AV}(T, Q) \mathrm{Z}$ calculations. However, the burden is larger since 4 calculations per geometry will be required. In summary, similar trends are expected at $\mathrm{CI}(\mathrm{Q})$ and other levels of theory. If one then adds the difficulty of using the 
Table 4 CPU times for raw HF, CI(Q), CCSD, CCSD(T) and extrapolated calculations with a d-AVXZ basis set (in seconds)

\begin{tabular}{|c|c|c|c|c|c|c|c|c|c|c|c|}
\hline \multirow[t]{2}{*}{$X$} & \multicolumn{3}{|l|}{$\mathrm{HF}$} & \multicolumn{3}{|l|}{$\mathrm{CI}(\mathrm{Q})^{\mathrm{a}}$} & \multicolumn{3}{|c|}{$\mathrm{CCSD}^{\mathrm{a}}$} & \multicolumn{2}{|c|}{$\operatorname{CCSD}(\mathrm{T})^{\mathrm{a}, \mathrm{b}}$} \\
\hline & $\mathrm{HeQ}$ & $\mathrm{He}_{2}$ & $\mathrm{CP}$ & HeQ & $\mathrm{He}_{2}$ & $\mathrm{CP}$ & $\mathrm{HeQ}$ & $\mathrm{He}_{2}$ & $\mathrm{CP}$ & $\mathrm{He}_{2}$ & $\mathrm{CP}$ \\
\hline \multicolumn{12}{|l|}{ Raw } \\
\hline$D$ & 0.0 & 0.1 & $0.1^{\mathrm{c}}$ & 0.1 & 0.1 & $0.2^{\mathrm{c}}$ & 0.1 & 0.1 & $0.2^{\mathrm{c}}$ & 0.1 & $0.2^{\mathrm{c}}$ \\
\hline$T$ & 0.4 & 0.6 & 1.0 & 0.9 & 0.7 & 1.6 & 1.0 & 0.7 & 1.7 & 0.9 & 1.9 \\
\hline$Q$ & 2.5 & 7.7 & 10.2 & 24.0 & 9.4 & 33.4 & 10.1 & 8.9 & 19.0 & 10.4 & 20.5 \\
\hline 5 & 34.5 & 83.9 & 118.4 & 216.4 & 92.1 & 308.5 & 100.2 & 91.0 & 191.2 & 99.5 & 199.7 \\
\hline 6 & 215.6 & 616.6 & 832.2 & $1,825.2$ & 916.3 & $2,741.5$ & 574.8 & 651.9 & $1,226.7$ & 696.3 & $1,271.1$ \\
\hline \multicolumn{12}{|c|}{ Extrapolated } \\
\hline$(T, Q)$ & 2.9 & 8.3 & 11.2 & 24.9 & 10.1 & 35.0 & 11.1 & 9.6 & 20.8 & 11.3 & 22.4 \\
\hline$(Q, 5)$ & 37.0 & 91.6 & 128.6 & 245.4 & 101.5 & 341.9 & 110.3 & 99.9 & 210.4 & 109.9 & 220.2 \\
\hline$(5,6)$ & 250.1 & 700.5 & 950.6 & $2,041.5$ & $1,008.4$ & $3,049.4$ & 675.0 & 742.9 & $1,418.0$ & 795.8 & $1,470.8$ \\
\hline
\end{tabular}

a All CPU times refer to the first calculated point and include the time spent in the restricted HF calculation

b Obviously, no triple excitations can be observed for HeQ, with the CISD and CCSD results corresponding to a FCI calculation. The HeQ column is therefore missing and should be taken as the corresponding column for a CCSD calculation

${ }^{c}$ Although taken here as the sum of the $\mathrm{He}_{2}$ and $\mathrm{HeQ} \mathrm{CPU}$ times, for an $\mathrm{AB}$ (heteronuclear) species the times for $\mathrm{CP}$ correspond to the sum of the $\mathrm{AB}, \mathrm{AQ}$ and $\mathrm{BQ}$ ones

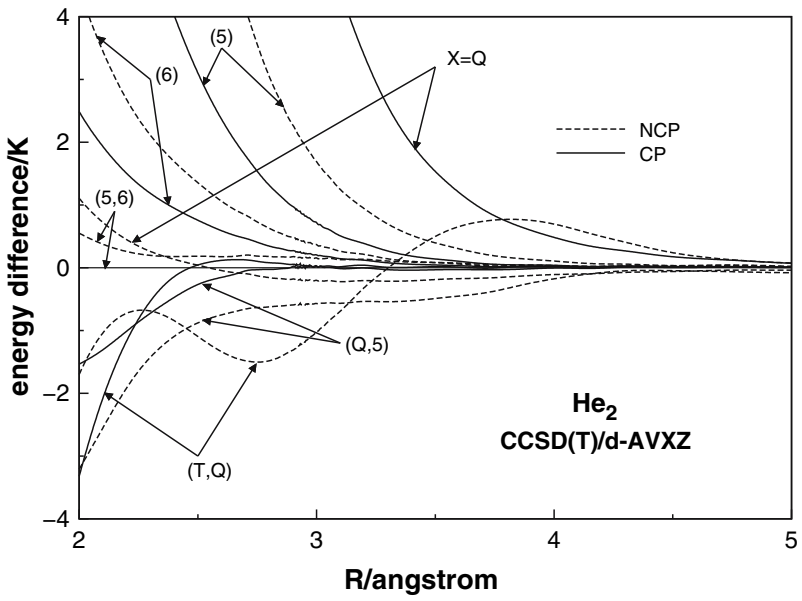

Fig. 4 Deviations of the raw d-AVTZ, d-AVQZ, and d-AV5Z raw $\operatorname{CCSD}(T)$ interaction energies with respect to the extrapolated d-AV $(5,6) Z$ curve with CP. Also shown are the deviations for the $\mathrm{d}-\mathrm{AV}(T, Q) \mathrm{Z}, \mathrm{d}-\mathrm{AV}(Q, 5) \mathrm{Z}$, and d-AV $(5,6) \mathrm{Z}$ interactions both with $\mathrm{NCP}$ and $\mathrm{CP}$. Although perceivable, the noise of typically $1 \mathrm{cK}$ (in a few points even up to $2 \mathrm{cK}$ ) in the densely covered regions has been partly removed by connecting the calculated points with smooth splines

$\mathrm{CP}$ method for open-shell interactions (where the electronic state of the fragments - often more than two-in a polyatomic system can be even hardly defined a priori except for an infinite separation), one concludes that CBS extrapolation can be a viable general alternative (or, if used jointly, an asset for quality) to counterpoise.

\section{Concluding remarks}

We have reported calculations on the helium dimer using the conventional $\mathrm{CI}(\mathrm{Q}), \mathrm{CCSD}$, and $\operatorname{CCSD}(\mathrm{T})$ methods with various orbital basis sets of the $\mathrm{d}-\mathrm{AV} X \mathrm{Z}$ type. The results have been extrapolated to the one electron basis set limit both using the $\mathrm{CP}$ method and the traditional NCP one. A generalized uniform singlet- and triplet-pair extrapolation method has been employed for the extrapolation procedure. After introduction of appropriate corrections to mimic FCI, the energies were predicted to be in very good agreement with the best available estimates. Two other findings are worth mentioning. First, the CP correction has been found invariant to size-consistency within the accuracy of the calculations, i.e., the $\mathrm{CP}$ correction is the same for $\mathrm{CI}$ and $\operatorname{CCSD}(\mathrm{T})$. This observation applies also to the extrapolated results once the numerical noise is considered. To our knowledge, this has not been reported before. Second, the results have also shown that extrapolation to the CBS limit can offer an alternative to the counterpoise correction that yields a more accurate potential energy while being more economical. It may therefore help on overcoming the difficulties with BSSE, particularly when more than two fragments are present. This will not contradict previous findings [7] since relatively large basis sets and cardinal numbers may be required for accurate predictions. In fact, the present work may provide a hint on the basis set size required to achieve reliable results for other systems. Of course, further studies that may test the generality of the present approach would be valuable, particularly for open-shell interactions given the utmost importance of having accurate potentials for reaction dynamics.

Acknowledgments This work has the support of Fundação para a Ciência e a Tecnologia, Portugal (Contracts POCI/QUI/60501/2004, POCI/AMB/60261/2004, and REEQ/128/QUI/2005), under the auspices of POCI 2010 of Quadro Comunitário de Apoio III co-financed by FEDER. 


\section{References}

1. Pople JA, Binkley JS, Seeger R (1976) Int J Quant Chem Symp 10:1

2. Pople JA (1999) Rev Mod Phys 71:1267

3. Bartlett RJ, Purvis GDIII (1980) Phys Scr 21:255

4. Helgaker T, Jørgensen P, Olsen J (2000) Molecular electronicstructure theory. Wiley, Chichester

5. Gdanitz RJ, Ahlrichs R (1988) Chem Phys Lett 143:413

6. Chalasinski G, Gutowski M (1988) Chem Rev 88:943

7. Halkier A, Klopper W, Helgaker T, Jørgensen P, Taylor PR (1999) J Chem Phys 111:9157

8. Lee JS, Park SY (2000) J Chem Phys 112:10746

9. Dunning TH Jr (2000) J Phys Chem A 104:9062

10. Brutschy B, Hobza P (eds.) (2000) Chem Rev 100:3861

11. Barrientos C, Sordo JA (2007) Theor Chim Acta 118:733

12. Boys F, Bernardi F (1970) Mol Phys 19:553

13. Johansson A, Kollman P, Rothenberg S (1973) Theor Chim Acta 29:167

14. Daudey JP, Claverie P, Malrieu JP (1974) Int J Q Chem 1:8

15. Wells BH, Wilson S (1983) Chem Phys Lett 101:429

16. White JC, Davidson E (1990) J Chem Phys 93:8029

17. Valiron P, Mayer I (1997) Chem Phys Lett 275:46

18. Mierzwicki K, Latajka Z (2000) Chem Phys Lett 325:465

19. Murrell JN, Carter S, Farantos SC, Huxley P, Varandas AJC (1984) Molecular potential energy functions. Wiley, Chichester

20. Varandas AJC (2007) J Chem Phys 126:244105

21. Schwenke DW, Truhlar DG (1984) J Chem Phys 82:2418

22. Dunning TH Jr (1989) J Chem Phys 90:1007

23. Dunning TH Jr, Peterson KA, Woon DE (1998) Schleyer PvR, Allinger NL, Clark T, Gesteiger J, Kolman PA, Schaefer HF III (eds) In: Encyclopedia of computational chemistry. Wiley, Chichester, $\mathrm{p} 88$

24. Gdanitz RJ (1999) Mol Phys 96:1423

25. van de Bovenkamp J, Van Duijneveldt FB (1999) J Chem Phys 110:11141

26. van Mourik T, Dunning TH Jr (1999) J Chem Phys 111:9248

27. Klopper W, Noga J (1995) J Chem Phys 103:6127

28. Noga J, Klopper W, Kutzelnigg W (1997) In: Bartlett RJ (ed) Recent advances in coupled-cluster Methods. World Scientific, Singapore, $\mathrm{p} 1$

29. Anderson JB (2001) J Chem Phys 115:4546

30. Anderson JB (2004) J Chem Phys 120:9886
31. Korona T, Williams H, Bukowski R, Jeziorski B, Szalewicz K (1997) J Chem Phys 106:5109

32. Klopper W (2001) J Chem Phys 115:761

33. Bartlett R (1995) In: Yarkony D (ed) Modern electronic structure theory. World Scientific, Singapore

34. Raghavachari K, Trucks GW, Pople JA, Head-Gordon M (1989) Chem Phys Lett 115:479

35. Komasa J (1999) J Chem Phys 110:7909

36. Komasa J (2001) J Chem Phys 115:158

37. Cencek W, Jeziorska M, Bukowski R, Jaszuński M, Jeziorski B, Szalewicz K (2004) J Phys Chem A 108:3211

38. Patkowski K, Cencek W, Jeziorka M, Jeziorski B, Szalewicz K (2007) J Phys Chem A 111:7611

39. Feller D, Sordo JA (2000) J Chem Phys 113:485

40. Schwartz C (1962) Phys Rev 126:1015

41. Kutzelnigg W, Morgan JD III (1992) J Chem Phys 96:4484

42. Flores JR, Slupski R, Jankowski K (2006) J Chem Phys 124: 104107

43. Helgaker T, Klopper W, Koch H, Noga J (1997) J Chem Phys 106: 9639

44. Feller D, Peterson KA, Crawford TD (2006) J Chem Phys 124: 054107

45. Varandas AJC (2000) J Chem Phys 113:8880

46. Varandas AJC (2007) Phys Scr (Comm At Opt Mol Phys) 76:C28

47. Bytautas L, Ruedenberg K (2005) J Chem Phys 122:154110

48. Varandas AJC (2007) J Chem Phys 127:114316

49. Truhlar DG (1998) Chem Phys Lett 294:45

50. Varandas AJC (2008) J Phys Chem A 112:1841-1850

51. Karton A, Martin JML (2006) Theor Chem Acc 115:330

52. Varandas AJC, Piecuch P (2006) Chem Phys Lett 430:448

53. Varandas AJC (2007) Chem Phys Lett 443:398

54. Werner H-J, Knowles PJ, Lindh R, Schütz M, Celani P, Korona T, Manby FR, Rauhut G, Amos RD, Bernhardsson A, Berning A, Cooper DL, Deegan MJO, Dobbyn AJ, Eckert F Hampel C, Hetzer G, Lloyd AW, McNicholas, SJ, Meyer W, Mura ME, Nicklass A, Palmieri P, Pitzer R, Schumann U, Stoll H, Stone AJ, Tarroni R, Thorsteinsson T (2003). Molpro, version 2002.6, a package of ab initio programs

55. Kobus J (1993) Chem Phys Lett 202:7

56. Gdanitz RJ (2000) J Chem Phys 113:5145

57. Jeziorka M, Cencek W, Patkowski K, Jeziorski B, Szalewicz K (2007) J Chem Phys 127:124303 